TITLE:

\title{
Mechanism of interconversion among radiation-induced defects in amorphous silicon dioxide
}

$\operatorname{AUTHOR}(S)$ :

Uchino, T; Takahashi, M; Yoko, T

CITATION:

Uchino, T ...[et al]. Mechanism of interconversion among radiation-induced defects in amorphous silicon dioxide. PHYSICAL REVIEW LETTERS 2001, 86(9): 1777-1780

ISSUE DATE:

2001-02-26

URL:

http://hdl.handle.net/2433/50392

RIGHT:

Copyright 2001 American Physical Society 


\title{
Mechanism of Interconversion among Radiation-Induced Defects in Amorphous Silicon Dioxide
}

\author{
Takashi Uchino,* Masahide Takahashi, and Toshinobu Yoko \\ Institute for Chemical Research, Kyoto University, Uji, Kyoto 611-0011, Japan
}

(Received 12 July 2000)

\begin{abstract}
We here present a series of $a b$ initio quantum-chemical calculations on clusters of atoms modeling several oxygen-deficiency-related defects in amorphous silica and illustrate how these defect centers will change their atomic configurations upon photoionization. We first give theoretical evidence that structural conversion from a neutral oxygen monovacancy to a divalent $\mathrm{Si}$ defect is possible, explaining the observed photoluminescence properties associated with these defects.
\end{abstract}

DOI: 10.1103/PhysRevLett.86.1777

Silicon dioxide is not only one of the most abundant materials on earth but also a material of considerable technological importance, including its use in microelectronic devices and optical fiber communications [1]. Furthermore, since the discovery of photoinduced refractive index changes in Ge-doped amorphous silicon dioxide [2], the finding has opened up the potential of a wide variety of applications in communication and sensing technology such as photoinduced Bragg gratings [3,4]. It is well accepted that the photosensitivity of amorphous silicon dioxide $\left(a-\mathrm{SiO}_{2}\right)$ is closely related to the nature of intrinsic point defects that can be transformed into other defect species during the irradiation process [5]. Among other defect centers in $a-\mathrm{SiO}_{2}$, oxygen-deficiency centers (ODCs) have attracted much interest since they are believed to play a vital role in the irradiation process and subsequent photostructural changes [5,6]. It has been demonstrated that there exist at least two distinct diamagnetic ODCs in $a-\mathrm{SiO}_{2}$; these are commonly denoted as ODC(I) and ODC(II), giving rise to the photoabsorption bands at $\sim 7.6$ and $\sim 5.0 \mathrm{eV}$, respectively [6,7]. It should also be noted that these ODCs are transformed into the so-called $E^{\prime}$ center upon strongly ionizing radiation with, for example, $\operatorname{ArF}[7-9]$ and $\mathrm{KrF}[8,10,11]$ excimer lasers. The generic term " $E^{\prime}$ center [12]" is commonly used for different paramagnetic variants comprising an unpaired spin in an $s p^{3}$-like orbital of a three-coordinated $\mathrm{Si}$ atom, namely, $\equiv \mathrm{Si} \bullet(\equiv$ and $\bullet$ represent three $\mathrm{Si}-\mathrm{O}$ bonds and an unpaired electron, respectively).

Although the physical origin of the diamagnetic ODCs is still a subject of controversy, there is a general consensus that the "relaxed oxygen vacancy," namely, the $\equiv \mathrm{Si}-\mathrm{Si} \equiv$ bond having a $\mathrm{Si}-\mathrm{Si}$ bond distance identical with that of $\mathrm{H}_{3} \mathrm{Si}-\mathrm{SiH}_{3}(\sim 2.3 \AA)$, is a good candidate for ODC(I) [6,7]. As for ODC(II), however, there are at least two alternatives. One is the "unrelaxed oxygen monovacancy," namely, the $\equiv \mathrm{Si} \cdots \mathrm{Si} \equiv$ bond having a $\mathrm{Si} \cdot \mathrm{Si}$ distance similar to that of regular $\mathrm{Si}-\mathrm{O}-\mathrm{Si}$ bonding $(\sim 3.1 \AA)$ [7], and the other is the "divalent $\mathrm{Si}$ " having two $\mathrm{Si}-\mathrm{O}$ bonds and a lone pair of electrons in a Si $s p^{2}$ hybrid orbital [13-15]. Recent theoretical calculations [16-19] have shown that the observed optical prop-
PACS numbers: 61.72.Ji, 61.43.Fs, 61.80.Ba, 68.35.Dv

erties of ODC(II) can be reproduced almost satisfactorily by the divalent $\mathrm{Si}$ model rather than the unrelaxed-oxygenvacancy model, suggesting that the latter model will not be responsible for the $\sim 5-\mathrm{eV}$ photoabsorption band [20]. It has also been found that a photoluminescence (PL) band at $\sim 4.4 \mathrm{eV}$ is observed when exciting a $\sim 5.0-\mathrm{eV}$ photoabsorption band in $a-\mathrm{SiO}_{2}$ [6]. This PL band most likely results from a singlet-to-singlet decay of the divalent $\mathrm{Si}$ defect [21]. Surprisingly, this typical emission feature of the divalent defect is also observed upon excitation of the relaxed oxygen vacancy in the $7-8 \mathrm{eV}$ region [22]. Several researchers $[6,14,23,24]$ have proposed that an interconversion may occur between the divalent defect and the relaxed oxygen vacancy upon ultraviolet irradiation, although a detailed mechanism of this interconversion is unknown.

To get a better knowledge about the formation mechanism among these preexisting and photoinduced defects in $a-\mathrm{SiO}_{2}$, we here employ ab initio quantum-chemical methods using clusters of atoms that model the local structure of several diamagnetic and paramagnetic defect centers. It has been demonstrated that $a b$ initio quantum-chemical approaches are useful to investigate the structure and vibrational properties of liquids as well as amorphous solids [25-28]. In particular, the defect states in disordered solids are, in general, quite localized, indicating that their structure and energy states will be reasonably modeled by such cluster calculations [5].

In this work, we, hence, employ the relaxed oxygen vacancy and the divalent Si defect as models of ODC(I) and ODC(II), respectively. Previous quantum-chemical calculations, especially on the divalent Si defect, employed rather small clusters modeling solely the corresponding defect sites [16-18]. However, the photoinduced processes will involve more remote spheres of coordination environment of the defects of interest and utilize structural freedom in the nondense-packed structure of amorphous systems. Thus, we use two types of relatively large isomers, $\mathrm{Si}_{15} \mathrm{O}_{17} \mathrm{H}_{24}$, modeling the local configurations of the relaxed oxygen vacancy [model 1, see Fig. 1(a)] and the divalent Si [model 2, see Fig. 1(b)] embedded in the silica framework. The "surface" silicon atoms of these clusters were terminated by $\mathrm{H}$ atoms, which is a 

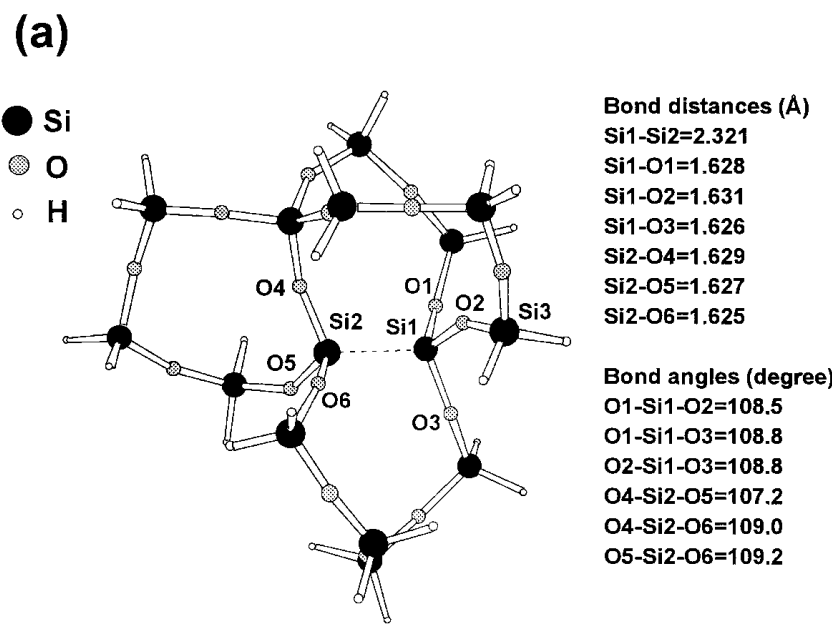

(b)

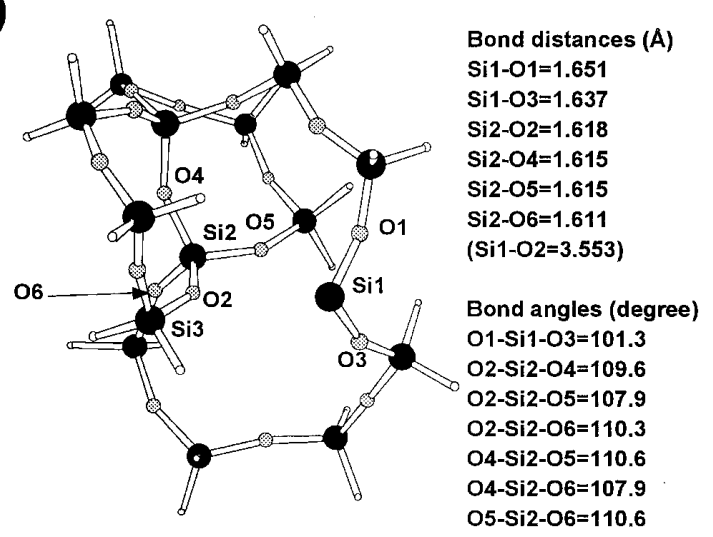

FIG. 1. Optimized geometries of the $\mathrm{Si}_{15} \mathrm{O}_{17} \mathrm{H}_{24}$ clusters calculated at the HF/6-31G(d) level: (a) the relaxed oxygen vacancy model (model 1), (b) the divalent Si model (model 2). Principal bond distances and bond angles are shown in $\AA$ and degrees, respectively.

commonly used technique to saturate the cluster dangling bonds [16-19,25,26]. The geometries of these clusters were fully optimized at the Hartree-Fock (HF) level using the 6-31G $(d)$ basis set [29]. We then reoptimized the geometries of the above two clusters by assuming a total charge of +1 to simulate the photoinduced ionization process. The positively charged clusters derived from models 1 and 2 are referred to as models $1(+)$ and $2(+)$, respectively (see also Fig. 2). The equilibrium geometries of these open shell systems were obtained at the unrestricted open HF (UHF) level with the 6-31G $(d)$ basis set, without imposing any structural constraints. All ab initio MO calculations in this Letter have been performed with the GAUSSIAN-98 program [30].

The HF/6-31G $(d)$ geometries of models 1 and 2 are shown in Fig. 1. The $\mathrm{Si}-\mathrm{Si}$ bond distance of the oxygen defect site model 1 is calculated to be $2.321 \AA$, which is in good agreement with the values reported previously for a smaller $(\mathrm{OH})_{3} \mathrm{Si}-\mathrm{Si}(\mathrm{OH})_{3}$ cluster $[18,31](2.30-2.32 \AA)$ modeling ODC(I). We have also found that the $\dot{\mathrm{S}} \mathrm{i}-\mathrm{O}$
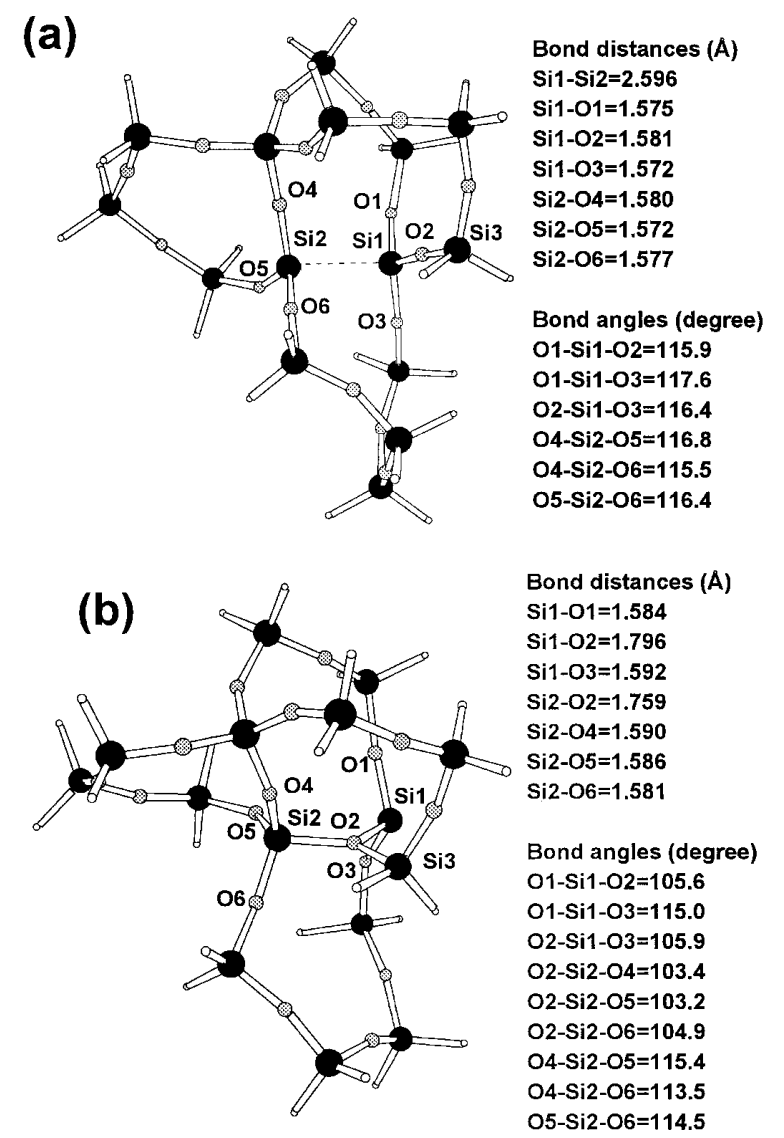

FIG. 2. Optimized geometries of the $\left(\mathrm{Si}_{15} \mathrm{O}_{17} \mathrm{H}_{24}\right)^{+}$clusters calculated at the UHF/6-31G $(d)$ level: (a) model $1(+)$, (b) model $2(+)$. Principal bond distances and bond angles are shown in $\AA$ and degrees, respectively.

bond distances (1.637 and $1.651 \AA$ ) and the $\mathrm{O}-\ddot{\mathrm{S}} \mathrm{i}-\mathrm{O}$ angle $\left(101.3^{\circ}\right)$ in model $2(\bullet \bullet$ indicates a lone pair of electrons on the divalent defect) agree well with the corresponding bond distances $(\sim 1.64-\sim 1.65 \AA)$ and the bond angle $\left(\sim 101^{\circ}\right)$ calculated for a smaller $\mathrm{Si}\left(\mathrm{OSiH}_{3}\right)_{2}$ cluster $[16,17]$ modeling ODC(II). These results suggest that, as far as the local structural parameters are concerned, these neutral oxygen vacancies are hardly affected by the surrounding environments.

Table I shows the Mulliken atomic charges for the Si atoms associated with the defect sites in the model clusters. We see from Table I that the atomic charge, $q$, of the divalent $\mathrm{Si}$ defect $(\mathrm{Si} 1)$ in model $2\left(q_{\mathrm{Si} 1}=0.836\right)$ is about

TABLE I. Calculated Mulliken atomic charges, $q$, and atomic spin densities, $\rho$, for the $\mathrm{Si}$ atoms associated with the defect centers in the model clusters.

\begin{tabular}{ccccccc}
\hline \hline & Model 1 & Model 2 & \multicolumn{2}{c}{ Model } & $1(+)$ & \multicolumn{2}{c}{ Model 2(+) } \\
& $q$ & $q$ & $q$ & $\rho$ & $q$ & $\rho$ \\
\hline Si1 & 1.293 & 0.836 & 1.388 & 0.402 & 1.321 & 0.912 \\
Si2 & 1.290 & 1.658 & 1.380 & 0.411 & 1.706 & 0.005 \\
\hline \hline
\end{tabular}


half the atomic charge of the ordinary four-coordinated $\mathrm{Si}$ atom $(\mathrm{Si} 2)$ in the same model $\left(q_{\mathrm{Si} 2}=1.658\right)$. Table I also shows that the atomic charge of the three-coordinated $\mathrm{Si}$ atoms in model $1\left(q_{\mathrm{Si} 1, \mathrm{Si} 2} \sim 1.29\right)$ is nearly three quarters of that of the four-coordinated $\mathrm{Si}(\mathrm{Si} 2)$ in model 2. These results elucidate that the $\mathrm{Si}$ atomic charge is almost linearly correlated to the number of $\mathrm{Si}-\mathrm{O}$ bonds in the respective $\mathrm{SiO}_{n}(n=2,3,4)$ units.

Since models 1 and 2 have the same stoichiometry of $\mathrm{Si}_{15} \mathrm{O}_{17} \mathrm{H}_{24}$ it is interesting to compare their total energies. It has been found that model 1 is lower than model 2 in total energy by $1.18 \mathrm{eV}$. This calculated value is in agreement with the observed enthalpy difference between ODC(I) and ODC(II), $1.0 \mathrm{eV}$ [7], obtained from a fictive temperature dependence of the $\sim 4$.4-eV photoluminescence band due to ODC(II). Thus, we consider that the present clusters most likely represent the fundamental features of two types of ODCs in the condensed environments and can be used as reasonable models of the ODCs in actual $a-\mathrm{SiO}_{2}$.

The optimized geometry of model $1(+)$ calculated at the UHF/6-31G $(d)$ level is shown in Fig. 2(a). The spin density of the unpaired electron in model $1(+)$ is almost equally distributed between $\mathrm{Si} 1$ and $\mathrm{Si} 2$, giving rise to similar atomic spin densities $\rho$ for the two Si atoms ( $\rho_{\mathrm{Si} 1}$, $\rho_{\mathrm{Si} 2} \sim 0.40$, see Table I) [32]. The resultant $\mathrm{Si}-\mathrm{Si}$ bond distance for the defect is $2.596 \AA$, which is slightly longer than the corresponding bond distance $(2.321 \AA)$ calculated for model 1. Figure 2(b) shows the UHF/6-31G $(d)$ geometry of model $2(+)$. One notices from Fig. 2(b) that the Sil atom in model $2(+)$ attracts a nearby oxygen atom (O2), forming a three-coordinated $\mathrm{Si}$ (Si1) and a three-coordinated $\mathrm{O}(\mathrm{O} 2)$ atom. It should be worth mentioning that the spin density of the unpaired electron in model $2(+)$ is mainly located on $\operatorname{Si} 1\left(\rho_{\mathrm{Si} 1}=0.912\right)$. The average $\mathrm{O}-\mathrm{Si} 1-\mathrm{O}$ bond angle in model $2(+)$ was calculated to be $108.8^{\circ}$, indicating that the valence orbitals of Sil are characterized by the $s p^{3}$-like hybrid orbitals. These calculated results allow us to suggest that, when one of the lone pairs of electrons on the divalent Si defect is excited to the conduction band, the silicon $s p^{2}$ hybrid orbitals on the two-coordinated center will be relaxed into the $s p^{3}$-like ones by attracting a nearby bridging oxygen atom, resulting in the three-coordinated Si site having an unpaired spin. Accordingly, the three-coordinated Si (Si1) in model $2(+)\left(q_{\mathrm{Si} 1}=1.321\right)$ becomes more positive than the two-coordinated $\mathrm{Si}(\mathrm{Si} 1)$ in model $2\left(q_{\mathrm{Si} 1}=0.836\right)$. It should also be noted that the atomic charge of Si2 adjacent to Si1 in model $2(+)\left(q_{\mathrm{Si} 2}=1.706\right)$ is even larger than that of the corresponding atom in model $2\left(q_{\mathrm{Si} 2}=1.658\right)$, demonstrating that the nominal positive charge of model $2(+)$ is not localized strictly on the defect site but will be partially distributed over the surrounding $\mathrm{SiO}_{4}$ units [33].

It should be noted that the total energy of model $2(+)$ has been found to be lower than that of model $1(+)$ by $0.861 \mathrm{eV}$. This implies that the defect configuration shown in model $1(+)$ corresponds to a metastable mini-

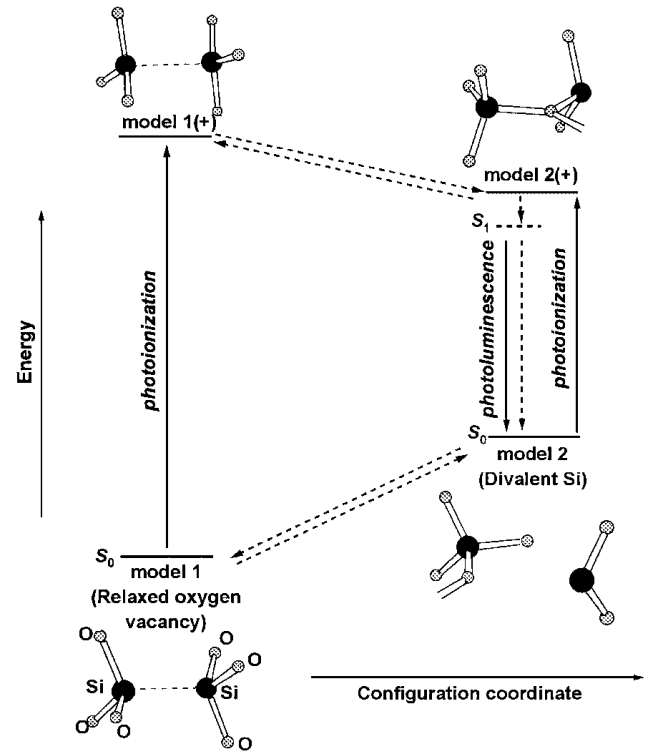

FIG. 3. Schematic illustration of the proposed interconversion mechanism among intrinsic and radiation-induced defects in amorphous silicon dioxide. The assumed energy level for the first excited singlet state $\left(S_{1}\right)$ of the divalent $\mathrm{Si}$ defect is also shown for convenience. The broken arrows stand for nonradiative processes such as thermal transitions and recombinations.

mum and is possible to relax into a lower energy configuration shown in Fig. 2(b) during thermal processes. We see from Fig. 2 that this transformation is accomplished just by forming a new bond between $\mathrm{Si} 2$ and $\mathrm{O} 2$ at the expense of the $\mathrm{Si} 1-\mathrm{Si} 2$ bond. We suggest that the observed photoluminescence properties of $a-\mathrm{SiO}_{2}$ mentioned earlier is related to such a structural relaxation from model $1(+)$ to model $2(+)$ during irradiation process. Upon the $\sim 7.6-\mathrm{eV}$ irradiation, the valence electron in the relaxed oxygen vacancy will be excited, and, accordingly, a photoinduced defect center analogous to model $1(+)$ will be initially formed. This photoinduced center will then be (partially) relaxed into lower energy configurations such as model $2(+)$ through thermal processes. When an electron is trapped by model $2(+)$, the defect will decay into a divalent $\mathrm{Si}$ defect via its excited singlet state. It is most likely that this recombination gives rise to the singlet-to-singlet PL band at $\sim 4.4 \mathrm{eV}$, similar to the case of the direct excitation of a $5.0-\mathrm{eV}$ band due to the divalent Si defect.

The present formation and decay mechanisms of the photoinduced defects are summarized in the schematic energy level diagram shown in Fig. 3. It is likely that these mechanisms are responsible for the interconversions between relaxed oxygen vacancies and divalent $\mathrm{Si}$ defects preceded by excitation in the $\sim 7.6 \mathrm{eV}$ absorption band, and the observed PL properties of $a-\mathrm{SiO}_{2}$ can be reasonably interpreted in terms of this scheme.

We thank the Supercomputer Laboratory, Institute for Chemical Research, Kyoto University, for providing the 
computer time to use the CRAY T-94/4128 supercomputer. We also thank the Ministry of Education, Science, Sports and Culture, Japan, for the Grant-in-Aids for COE Research on Elements Science, No. 12CE2005.

*Electronic address: uchino@scl.kyoto-u.ac.jp

[1] E. H. Nicollian and J. R. Brews, MOS (Metal Oxide Semiconductor) Physics and Technology (Wiley, New York, 1982).

[2] K. O. Hill, Y. Fujii, D. C. Johnson, and B.S. Kawasaki, Appl. Phys. Lett. 32, 647 (1978).

[3] A. Othonos and K. Kalli, Fiber Bragg Gratings: Fundamentals and Applications in Telecommunications and Sensing (Artech House, Boston, 1999).

[4] R. Kashyap, Fiber Bragg Gratings (Academic, New York, 1999).

[5] A.H. Edwards, in Defects in Glasses, edited by F.L. Galeener, D.L. Griscom, and M. J. Weber (Materials Research Society, Pittsburgh, 1986), p. 3.

[6] L. Skuja, J. Non-Cryst. Solids 239, 16 (1998).

[7] H. Imai, K. Arai, and H. Imagawa, Phys. Rev. B 38, 12772 (1988).

[8] J.H. Stathis and M.A. Kastner, Phys. Rev. B 29, 7079 (1984).

[9] M. Rothschild, D. J. Ehrlich, and D. C. Shaver, Appl. Phys. Lett. 55, 1276 (1989).

[10] E. V. Amosov et al., Sov. J. Glass Phys. Chem. 18, 62 (1992).

[11] T.E. Tsai, E.J. Friebele, M. Rajaram, and S. Mukhapadhyay, Appl. Phys. Lett. 64, 1481 (1994).

[12] R. A. Weeks, J. Appl. Phys. 27, 1376 (1956).

[13] A. R. Silin and L. N. Skuja, J. Non-Cryst. Solids 71, 443 (1985).

[14] K. Awazu, H. Kawazoe, and K. Muta, J. Appl. Phys. 70, 69 (1991).

[15] L. Skuja, J. Non-Cryst. Solids 149, 77 (1992).

[16] B.L. Zhang and K. Raghavachari, Phys. Rev. B 55, R15 993 (1997).

[17] B. B. Stefanov and K. Raghavachari, Appl. Phys. Lett. 71, 770 (1997).

[18] B. B. Stefanov and K. Raghavachari, Phys. Rev. B 56, 5035 (1997).

[19] G. Pacchioni and R. Ferrario, Phys. Rev. B 58, 6090 (1998).
[20] We have recently proposed that [T. Uchino et al., Phys. Rev. B 62, 2983 (2000)] a new type of defect centers, in which one of the two oxygen atoms in a two-membered silica ring is missing, is also responsible for the $\sim 5.0-\mathrm{eV}$ photoabsorption band. However, we concentrate our interest on the interconversion between divalent $\mathrm{Si}$ defects and neutral oxygen monovacancies..

[21] L. N. Skuja, A. N. Streletsky, and A. B. Pakovich, Solid State Commun. 50, 1069 (1984).

[22] A. N. Trukhin, L. N. Skuja, A. G. Boganov, and V. S. Rudenko, J. Non-Cryst. Solids 149, 96 (1992).

[23] H. Nishikawa, E. Wantanabe, D. Ito, and Y. Ohki, Phys. Rev. Lett. 72, 2101 (1994).

[24] R. Boscaino, M. Cannas, F. M. Gelardi, and M. Leone, J. Phys. Condens. Matter 11, 721 (1999).

[25] M. O'Keeffe and G. V. Gibbs, J. Chem. Phys. 81, 876 (1984).

[26] T. Uchino, M. Takahashi, and T. Yoko, Phys. Rev. Lett. 84, 1475 (2000).

[27] T. Uchino, Y. Tokuda, and T. Yoko, Phys. Rev. B 58, 5322 (1998).

[28] T. Uchino and T. Yoko, J. Chem. Phys. 108, 8130 (1998).

[29] M. S. Gordon, Chem. Phys. Lett. 76, 163 (1980), and references therein.

[30] M.J. Frisch et al., Gaussian 98, Revision A7 (Gaussian Inc., Pittsburgh, 1998).

[31] G. Pacchioni and A. Basile, J. Non-Cryst. Solids 254, 17 (1999).

[32] In this work, we did not obtain an asymmetric configuration of the charged vacancy defect having a paramagnetic $\equiv \mathrm{Si} \bullet$ unit and a puckered $\equiv \mathrm{Si}^{+}$unit, which is the model of the $E_{1}^{\prime}$ center in $\alpha$ quartz. This is probably because a bridging oxygen atom behind the defect site, which is responsible for the puckered configuration of the positive fragment, is missing in the present model. In $a-\mathrm{SiO}_{2}$, however, such a back-oxygen site that is strategically located in $\alpha$ quartz will not have a well-defined position or may even be effectively absent. Thus, we believe the present model is not far from realistic, as far as the charged defect in $a-\mathrm{SiO}_{2}$ is concerned.

[33] It should be noted that the defect site seen in model 2(+) can be otherwise viewed as a charged vacancy defect having a paramagnetic $\equiv(\mathrm{Si} 1) \bullet$ unit and a puckered $\equiv(\mathrm{Si} 2)^{+}$ unit. In this model, one of the oxygen atoms $(\mathrm{O} 2)$ in the $\equiv(\mathrm{Si} 1) \bullet$ unit is responsible for the puckered configuration of the $\equiv(\mathrm{Si} 2)^{+}$unit instead of the back oxygen in the $E_{1}^{\prime}$-center model. 